\title{
ANALYSIS OF POROUS STRUCTURE IN PLASMA-SPRAYED COATING
}

\author{
RAdKA JŮZKOVÁ ${ }^{1}$, PAVEl CTIBOR ${ }^{2}$ AND ViKTOR BENEŠ $\check{S}^{3}$ \\ ${ }^{1}$ Private College of Economic Studies, Ltd., Lindnerova 575/1, 18000 Prague 8-Libeň, Czech Republic, \\ ${ }^{2}$ Institute of Plasma Physics ASCR, Za Slovankou 3, 18221 Prague 8-Libeň, Czech Republic, ${ }^{3}$ Faculty of \\ Mathematics and Physics, Charles University, Sokolovská 83, 18600 Praha 8-Karlín, Czech Republic \\ e-mail: radka.juzkova@svses.cz, ctibor@ipp.cas.cz, benesv@karlin.mff.cuni.cz \\ (Accepted February 23, 2004)
}

\begin{abstract}
The paper is devoted to the quantitative analysis of the microstructure of a ceramic plasma-sprayed coating. The aim is to describe the size and spatial distribution of approximately convex pores. A whole 3D specimen was subsequently grinded to obtain serial sections of small distance. Using image analysis techniques the centroids and size of individual pores are detected and the obtained data analysed by means of summary spatial statistics. The tests of complete spatial randomness are performed using various $3 \mathrm{D}$ characteristics and important practical conclusions for the material fabrication procedure are obtained.
\end{abstract}

Keywords: ceramic coating, morphological, porosity, second order characteristics, spatial distribution, volume reconstruction.

\section{INTRODUCTION}

The analysis of the microstructure of ceramic plasma-sprayed coatings is of great importance (Ctibor et al., 2002; 2003). A typical specimen, sprayed in the Institute of Plasma Physics ASCR, Prague, Czech Republic, is investigated in this paper. Its microstructure forms pores of various shapes. In the analysis approximately convex pores are detected and measured, their size and spatial distribution is a subject of interest. For hard materials, usually a single section is available that is studied by means of stereological methods (Ohser and Mücklich, 2000). To avoid ill-posed stereological problems, with great laboratory effort, we obtained serial sections along the whole specimen. This approach enhances the quantitative analysis considerably, since three-dimensional (3D) distance and second order characteristics can be directly estimated. However preliminary image analysis of serial sections requires careful and tedious work. Also, a computer program for $3 \mathrm{D}$ pore reconstruction has been prepared. The choice of estimators of contact distribution, nearest neighbour distance distribution, $\mathrm{K}$ - and L-function is discussed in the literature (Ohser and Mücklich, 2000; Baddeley and Gill, 1997; Moller and Waagepetersen, 2003) and we contribute with our practical results. Standard statistical Monte Carlo tests are applied for complete spatial randomness and the alternative of mild clustering is recognised.

Porosity affects important functional properties of any thermal spray coating like corrosion resistance, cohesion under induced mechanical stress/strain, electric resistivity and permittivity and others.
The factors governing the porosity level and also some spatial porosity features (such as clustering, inhomogeneity) are given by plasma spray gun parameters combination. The detection of pores clustering means, for us, that more attention has to be paid to powder feeding (especially the diameter of the feeder nozzle vs. plasma stream diameter, feeding point position adjustment).

\section{MATERIAL}

Titanates, in general, form a wide and important group of electronic ceramics. A mixture of $\mathrm{MgTiO}_{3}$ and $\mathrm{CaTiO}_{3}$, with a $\mathrm{Mg}: \mathrm{Ca}$ ratio equal to $94: 6$, was plasma sprayed at the Institute of Plasma Physics ASCR, Prague, Czech Republic (Ctibor et al., 2002). It was proven that the microstructure of a selected material is representative enough to serve as a pilot material for studying the microstructure of ceramic plasma deposits in general. A sample was manufactured using a water-stabilised plasma spray system WSP ${ }^{\circledR}$ PAL 160 . The feedstock powder size was $63-125 \mu \mathrm{m}$, final coat thickness was about $1 \mathrm{~mm}$. Volume studied in present paper has dimensions $450 \times$ $350 \times 240 \mu \mathrm{m}$ and is situated far enough from a free surface and from the substrate to prevent material "edge effects" (Fig 1).

Lamellas (usually called splats) are results of spreading and solidification of droplets accelerated against the substrate. The lamellas are principal structural units, basic "bricks" of a microstructural architecture of the coatings, in general more 
important for coating properties than crystallites local orientation, grain boundaries or similar structural features.

The steel substrate covered by plasma-sprayed ceramic coating was cut by a low speed saw (Leco, USA) equipped with a diamond wheel to prevent structural modifications. A cross section of the substrate-coating system was mounted in a resin. Then, grinding was completed with a rough grain diamond paste. Subsequent polishing of individual planes for large area section imaging was performed manually using diamond polishing paste (Struers, Denmark).

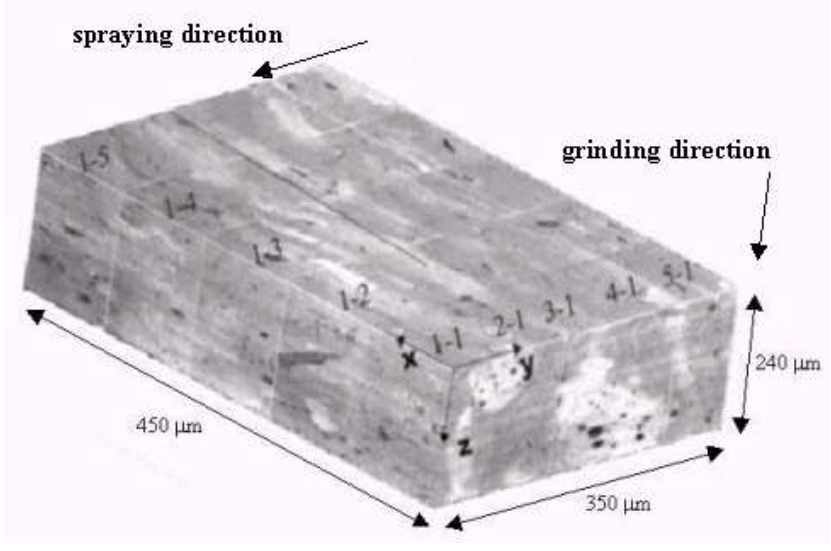

Fig. 1. The investigation of $3 D$ specimen of plasmasprayed ceramic coating. Eighty serial sections are obtained perpendicular to the grinding direction. Each section is a montage of $5 \times 5$ images observed by an optical microscope. The spraying direction is perpendicular to the substrate surface.

The creation of the large-area section involved a sequence of steps, namely:

1. Identification of a suitable large region on the first cross section plane covering 25 fields of view of the optical microscope, Neophot 32 (Zeiss, Germany), and RGB camera, TK-1070E (JVC, Japan).

2. Creation of a seamless "montage" of images covering all fields in this region of the first plane.

3. Physical removal of a small amount of material by polishing and identification of the same region on the second plane.

4. Creation of a seamless "montage" of all microstructural fields of view of the same region on the second plane.

5. Measurement of the distance between the subsequent planes by measuring the decrease in the sizes of pyramidal markers.
The resulting "montage" (cf. Tewari and Gokhale, 2000), was essentially a microstructural image of a large area (25 fields of view at $800 \times$ magnification in this case) having a high resolution $(\sim 0.13 \mu \mathrm{m} /$ pixel $)$. By repeating of the steps $1-5$ the stack of large area images is prepared. A combination of the resolution in one plane and the distance between planes $3 \mu \mathrm{m}$ was used with respect to the main structural features of plasma-sprayed coatings. Together, 80 aligned planes, each consisting of 25 high-resolution "montage" images were documented and all 2000 images stored in the computer to undergo the image analysis.

One large section after montage represents $450 \times$ $350 \times 240 \mu \mathrm{m}$, i.e. $3460 \times 2690$ pixels $\times 240 \mu \mathrm{m}$ (the $z$ axis represents 80 times removed $3 \mu \mathrm{m}$ by polishing).

\section{METHODS}

As described in the previous paragraph the whole three-dimensional (3D) specimen was cut in serial sections using grinding. The image of a section is two-dimensional (2D). The analysis of microstructural images was performed using automatic image analysis softwares Microimage (Olympus, 1999), and Lucia G3.52.

The image analysis performed on each single image consists of the following steps. Segmentation was done using the same threshold level for all pictures. It leads to the isolation of pores of all types (including both cracks and approximately convex pores). Using morphological opening (Serra, 1982) with spherical structural element other cracks than pores are deleted (Fig. 2). Another way based on erosion/reconstruction as described in Soille (1999), was not used since we believe that the selection induced by opening does not influence our statistics substantially. The reason is that pores do not contain much sharp corners of boundaries. Then, the parameters of pore sections are measured (centroid coordinates, area, perimeter, angle and length of maximum diameter).

In the second step, a computer program was prepared for the reconstruction of 3D pores from the section measurements. The centroid coordinates, number of intersected sections, minimum and maximum diameter and volume were estimated for each detected pore.

The aim of stereological methods used is to reconstruct 3D parameters of the microstructure from the measured parameters in 2D sections. Classical stereological methods (Ohser and Mücklich, 2000), are not needed because the stack of serial 
sections in fact yields 3D information directly. The spatial distribution is described by $3 \mathrm{D}$ contact distribution, nearest neighbour distribution, $K-$, $L-$ and the pair correlation function. Estimators of these characteristics are taken from Baddeley and Gill (1997), Moller and Waagepetersen (2003) and Ohser and Mücklich (2000). Special care should be given to the correction of edge effects. Comparing these estimated functions with those corresponding to a Poisson process the deviations from spatial randomness are classified, which is important for the description of the material studied.

Consider a convex 3D set $W$, a subset of the Euclidean space $R^{3}$. In our application, the centroids of pores in a specimen $W$, which is a rectangular parallelepiped (see Fig. 1), are observed. Let us denote the centroids $X_{1}, \ldots, X_{n}$, they are considered as a realisation of a homogeneous isotropic point process $\Phi$ as defined in Moller and Waagepetersen (2003). By $\Phi(B)$, the (random) number of points a set $B$ is denoted. The intensity $\lambda$ of such a process is the mean number of points in a unit volume, i.e. $\lambda=E \Phi\left([0,1]^{3}\right)$. An unbiased estimator of intensity is $\widehat{\lambda}=n / V(W)$, where $V(W)$ is the volume of $W$ and $n$ is a number of observed points. Another estimator for $\lambda^{2}$ (Ohser and Mücklich, 2000), is

$$
\widehat{\lambda^{2}}=\frac{n(n-1)}{(V(W))^{2}} .
$$

The distance between points $X_{i}, X_{j}$ is denoted

$$
d_{i j}=\left\|X_{i}-X_{j}\right\|
$$

where $\|$.$\| is the Euclidean norm. The window function$ $c_{W}$ is defined on $R^{3}$ as $c_{W}(h)=V(W \ominus\{o, h\})$, i.e. by morphological erosion of $W$ with a two point set $\{o, h\}, o$ being the origin of coordinate system. Its isotropised version on $R^{1}$ is

$$
\bar{c}_{W}(r)=\frac{1}{4 \pi} \int_{S^{2}} V(W \ominus\{o,(r, u)\}) d u,
$$

where $S^{2}$ is the unit sphere in $R^{3}$ and $(r, u)$ polar coordinates ( $r$ size, $u$ orientation) of a vector $h \in R^{3}$.

The $K$-function of $\Phi$ is defined as $\lambda K(r)=$ $E \Phi(b(o, r) \mid o \in \Phi)$, where on the right hand side there is the mean number of points in a ball $b(o, r)=\left\{x \in R^{3} ;\|x\| \leq r\right\}$ under the condition that $o$ is the point of the process $\Phi$. Since this condition has zero probability the Palm theory (Soille, 1999) is used for a rigorous definition. The relationship between $K$-function and the pair correlation function $\bar{g}$ is for $r \geq 0$

$$
K(r)=4 \pi \int_{0}^{r} x^{2} \bar{g}(x) d x .
$$

The $L$-function is just a normed version of $K$-function:

$$
L(r)=\left(\frac{3}{4 \pi} K(r)\right)^{\frac{1}{3}}, r \geq 0 .
$$

For the Poisson point process, it holds $L(r)=r, r \geq 0$.

An unbiased estimator of $K$-function is used from Ohser and Mücklich (2000):

$$
\widehat{K}(r)=\frac{2}{\widehat{\lambda^{2}}} \sum_{i=1}^{n-1} \sum_{j=i+1}^{n} \frac{1_{(0, r]}\left(d_{i j}\right)}{\bar{c}_{W}\left(d_{i j}\right)}, \quad r \geq 0 .
$$

For the rectangular parallelepiped $W$ with side lengths $a \leq b \leq c$, we have the window function

$$
\begin{aligned}
& \bar{c}_{W}(r)=a b c- \frac{2(a b+b c+a c)}{4} r+ \\
& \frac{2(a+b+c) r^{2}}{3 \pi}-\frac{r^{3}}{4 \pi}, \quad 0 \leq r \leq a .
\end{aligned}
$$

The estimator of $L$-function is obtained by plugging the estimator (3) of $K$-function into (2).

Let $D$ be the nearest neighbour distance, i.e. a random variable corresponding to the nearest distance from a given point of the process $\Phi$ to its neighbour. Its distribution function $D(r)=P(D \leq r)$ can be written as $\left(X_{i}\right.$ is any point of $\left.\Phi\right)$

$$
D(r)=1-P\left(\Phi\left(b\left(X_{i}, r\right)\right)=1\right), \quad r \geq 0 .
$$

Spherical contact distance $H$ can be defined as a distance of the nearest point of $\Phi$ from the origin. Its distribution function $H^{S}$ is

$$
H^{S}(r)=1-P(\Phi(b(o, r))=0), r \geq 0 .
$$

The point process is homogeneous Poisson if and only if it holds

$$
D(r)=H^{S}(r)=1-\exp \left\{-\lambda \frac{4 \pi}{3} r^{3}\right\}, r \geq 0 .
$$

The deviations from this theoretical form are used to recognise the interactions within the point process, either attractive or repulsive, possibly on different scales.

The clustering of the point process is observed in the graph for the distribution function of nearest neighbour distances when the curve is above the curve (Eq. 6) and in the graph for contact distribution function when the curve is below the curve (Eq. 6). 
In the opposite case, for distribution function of nearest neighbour distances below and for the contact distribution function above the curve (Eq. 6), we observe regularity in the point process.

Estimation of $D(r)$ and $H^{S}(r)$ is a problem because of edge effects, since for the points near to the boundary of $W$ the nearest neighbour need not be observed. Several methods for edge effect corrections are described in Baddeley and Gill (1997), Ohser and Mücklich (2000), Stoyan et al. (1995). We used the so called Kaplan-Meier estimators, see Baddeley and Gill (1997).

Let $d_{j}$ be the nearest neighbour distance of $X_{j} \in W$ and $\rho\left(X_{j}, \partial W\right)$ be the distance of $X_{j}$ to the boundary of the window $\mathrm{W}$ for each $j=1, \ldots n$. Then, the KaplanMeier estimator of $D(r)$ is

$\widehat{D}(r)=1-\prod_{s \leq r}\left(1-\frac{N\left\{j: d_{j}=s, d_{j} \leq \rho\left(X_{j}, \partial W\right)\right\}}{N\left\{j: d_{j} \geq s, \rho\left(X_{j}, \partial W\right) \geq s\right\}}\right)$,

where $r>0$, setting $0 / 0=0 . N$ denotes the number of points (cardinality) of the set.

To estimate the contact distribution function, a random or systematic point test set is used. We used a binomial point pattern $\left\{X_{1}^{t}, \ldots, X_{p}^{t}\right\}$ of $p$ random test points in $W$ and denote $d_{j}^{t}$ the contact distances of nearest point of the process in $W$ to the test point, $\rho\left(X_{j}^{t}, \partial W\right)$ denotes the distance of $X_{j}^{t}$ to the boundary of the window $\mathrm{W}$ for each $j=1, \ldots n$. Then, the Kaplan-Meier estimator $\widehat{H}^{S}$ used is

$\widehat{H}^{S}(r)=1-\prod_{s \leq r}\left(1-\frac{N\left\{j: d_{j}^{t}=s, d_{j}^{t} \leq \rho\left(X_{j}^{t}, \partial W\right)\right\}}{N\left\{j: d_{j}^{t} \geq s, \rho\left(X_{j}^{t}, \partial W\right) \geq s\right\}}\right)$

where $r>0$, setting $0 / 0=0 . N$ denotes the number of points (cardinality) of the set.

One of the main aims of the statistical analysis of measured data was to test the hypothesis of complete spatial randomness of convex pores within the specimen. Monte Carlo tests (Ohser and Mücklich, 2000) present a standard tool for this purpose. Nineteen independent realisations of the Poisson point process with the same intensity as that estimated from data are simulated. For each realisation the $K-, L-, H^{S}-, D$-functions are estimated (using Eqs. 3, 2, 8, 7, respectively) and their pointwise maximum and minimum is plotted. Using more realisations the bounds might be smoother, but as will be seen in the numerical results, the amount 19 is enough for our analysis. If the estimator of the same function obtained from data gets outside the plotted bounds for some $r$, we reject the null hypothesis of complete spatial randomness on a $95 \%$ confidence level at distance $r$.

\section{NUMERICAL RESULTS}

The microstructure of a ceramic plasma-sprayed deposit specimen of size $450 \times 350 \times 240 \mu \mathrm{m}$ was monitored on 80 parallel sections considered as equidistant with distance $3 \mu \mathrm{m}$. A typical result of morphological operations is in Fig. 2.
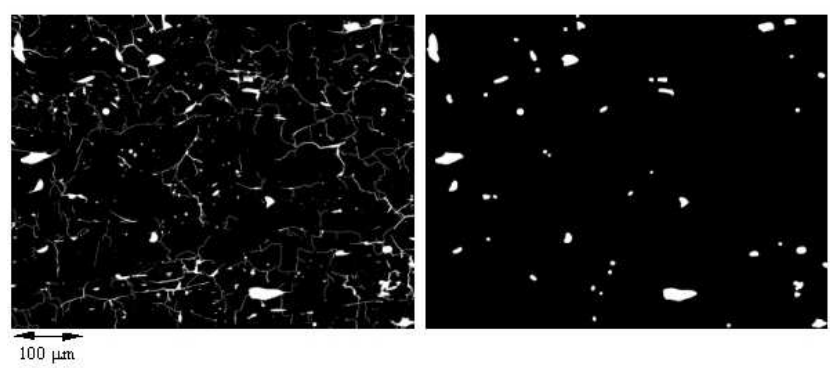

Fig. 2. The binary image of the $2 D$ microstructure in a section including pores and cracks (left) and the same microstructure after morphological opening (right).

The important pore volume histogram (Fig.3) is the starting point for further analysis, since from a physical point of view it is desirable to study pores of defined size classes. The pores with maximum diameter between 3-40 $\mu \mathrm{m}$ were detected. The mode of the distribution is about $4-6 \mu \mathrm{m}$.

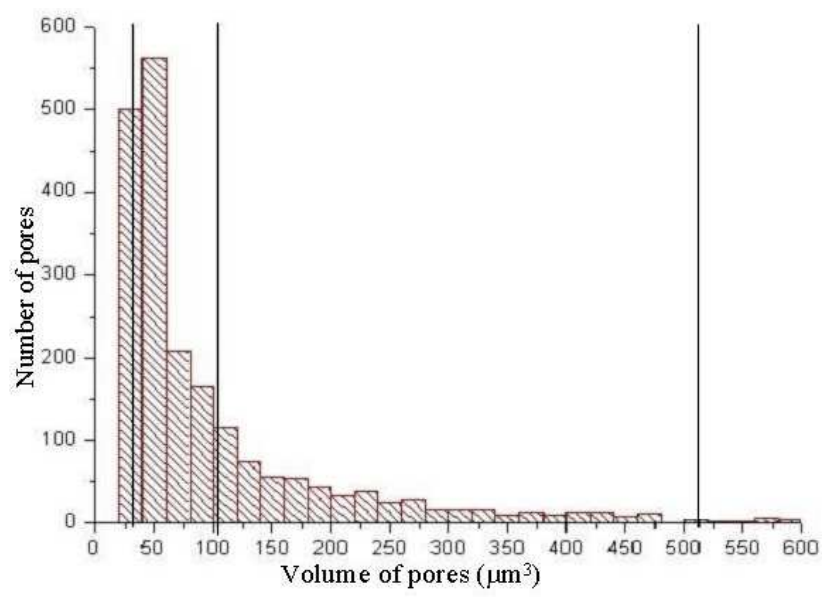

Fig. 3. Pore volume histogram; the volume of pores was estimated using Cavalieri method, i.e. $V=t \sum A_{i}$, where $t$ is the distance between sections and $A_{i}$ is the area corresponding to convex pores in $2 D$ images to $3 D$ pores. The vertical lines in histogram show limits of size categories (lines corresponding to diameters equal to 4, 6, 10 $\mu \mathrm{m}$ ) for a spherical shape model. 
The size categories were selected with respect to lamellae thickness, which is for this spraying technique and material typically given 3 to $6 \mu \mathrm{m}$. Interlamellar globular pores are usually smaller than $4 \mu \mathrm{m}$. Also, the smallest size class used $(4-6 \mu \mathrm{m})$ is supposed to correspond dominantly to pores between lamellas. Larger size class $(6-10 \mu \mathrm{m})$ is "intermediate", while the largest one $(10-20 \mu \mathrm{m})$ is supposed to correspond dominantly to voids in proximity of not well melted (i.e. not well flattened) lamellas. Also the different size classes could exhibit different behaviour. But, given the present state of the art, the above expressed opinion is not more than a hypothesis.

In Table 1, important size classes are defined with corresponding pore numbers in the sample corresponding to studied size classes.

Table 1. Number of pores in corresponding size categories

\begin{tabular}{|c|c|}
\hline Diameter of pores & Number of pores \\
\hline$\overline{\text { any }}$ & 2116 \\
\hline $4-20$ & 1601 \\
\hline $4-6$ & 598 \\
\hline $6-10$ & 548 \\
\hline $10-20$ & 455 \\
\hline
\end{tabular}

The experiment of plasma spraying, as described in the Materials section, may lead to hypoteses that there are some anisotropies or gradients in the spatial distribution of pores. A simple method to detect them is to plot projections of all pores onto coordinate planes. In Figs. 4-6 these plots, moreover, include information on pore size and no apparent phenomena of that kind are observed.

A finer analysis is based on summary statistics. In Figs. 7-9 the results are presented for different size classes, as suggested in Table 1. In each Figure the estimated $L-, H^{S}-$, and $D-$ functions are analysed. The argument $r$ of the functions corresponds to the distance. The starting value of $r$ in the graph is equal to the lower bound of diameters of pores in the studied size class because the distance between centroids of pores cannot be lower than the sum of their half diameters (e.g. the starting value of $r$ for the class 4$6 \mu \mathrm{m}$ is 4$)$. The value of $L$-function at the starting value of $r$ is equal to this value for the Poisson process and, therefore, the vertical axis of the graph starts at this $r$.
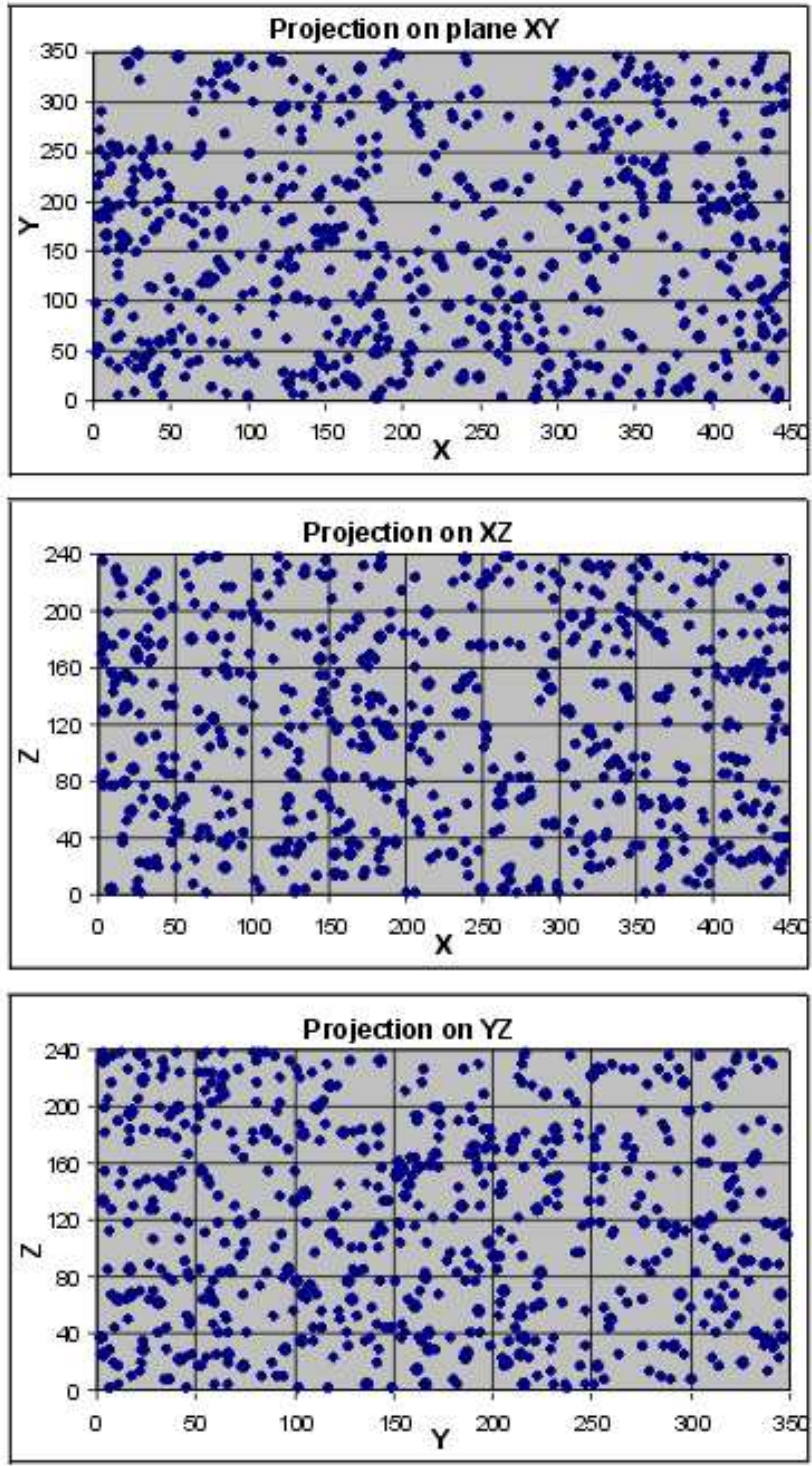

Fig. 4. Projection on the planes $X Y, X Z$ and $Y Z$ (see Fig. 1) for the pores of diameter equal to 4-6 $\mu \mathrm{m}$.

The rejection of the hypotesis of complete spatial randomnes is mostly apparent for $L$-function (in all three size classes) and for $D$-function (for smaller pores). Since these functions lie above the bounds for the Poisson process in some intervals, rejection tends towards clustering. For $H^{S}$-function, this corresponds to values below the bounds, which is apparent for the smallest size class and $27<r<40 \mu \mathrm{m}$.

Finally, if the whole set of events between 4-20 $\mu \mathrm{m}$ is evaluated, then since the smaller pores prevail, the null hypothesis is again rejected towards the alternative of clustering. 

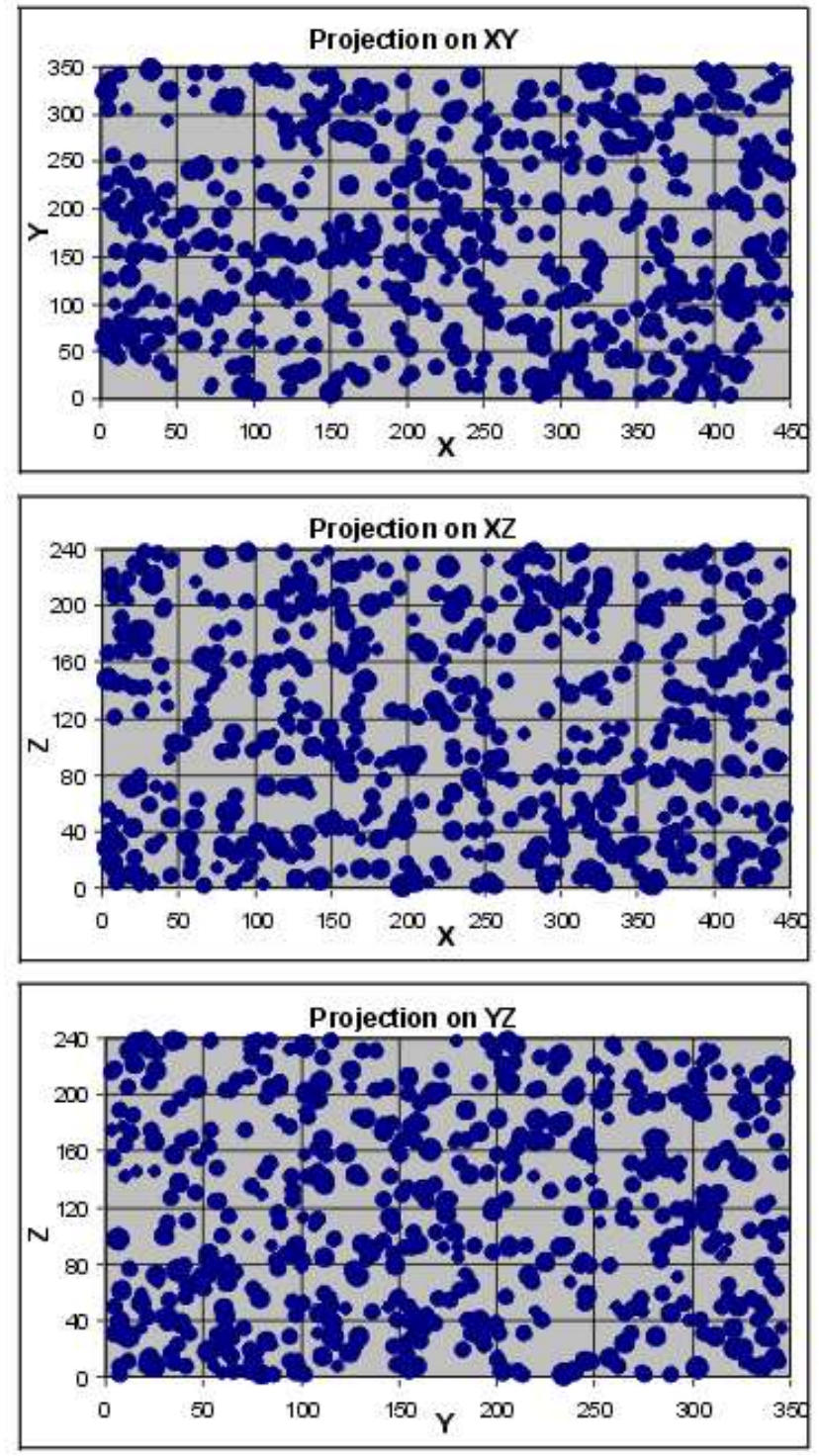

Fig. 5. Projection on the planes $X Y, X Z$ and $Y Z$ (see Fig. 1) for the pores of diameter equal to 6-10 $\mu \mathrm{m}$.

\section{CONCLUSIONS}

Globular pores represent one category of voids in plasma-sprayed coatings. These pores are distributed isotropically and their potential to deteriorate coating properties is proportional to their size. Moreover, all size categories of globular pores are prospective for the connection between the knowledge of the structure and the knowledge of behaviour of powder in the plasma. Spherical pores arise as defects in powder partly solidified before impacting on the substrate and as defects during spreading of impacting particles.
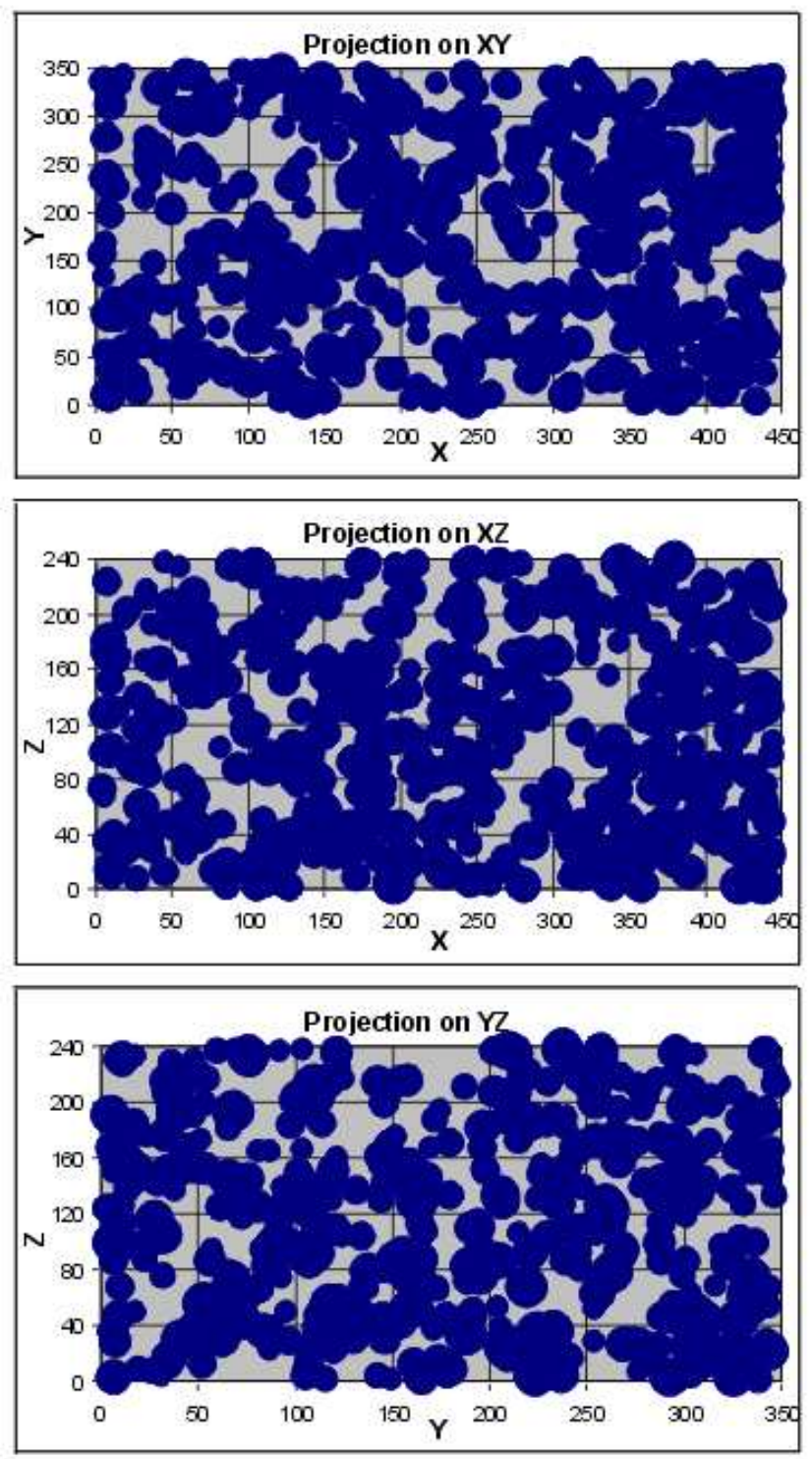

Fig. 6. Projection on the planes $X Y, X Z$ and $Y Z$ (see Fig. 1) for the pores of diameter equal to 10-20 $\mu \mathrm{m}$.

Plasma spraying is a very rapid process - in the setup used for the sample manufacturing studied approximately $6 \times 10^{5}$ powder particles are in $1 \mathrm{~cm}^{3}$ of plasma jet and travel together with them towards the substrate surface by velocity of hundreds of $\mathrm{m} / \mathrm{s}$. It means that each $0.001 \mathrm{~s}$ new $1.8 \times 10^{5}$ particles are in the same volume. Particles are mixed with plasma which burns in the air. From the thermal point of view plasma gives to the particles energy for complete melting before access of the substrate. From the kinetic point of view plasma gives energy for spreading of particles on the substrate. 

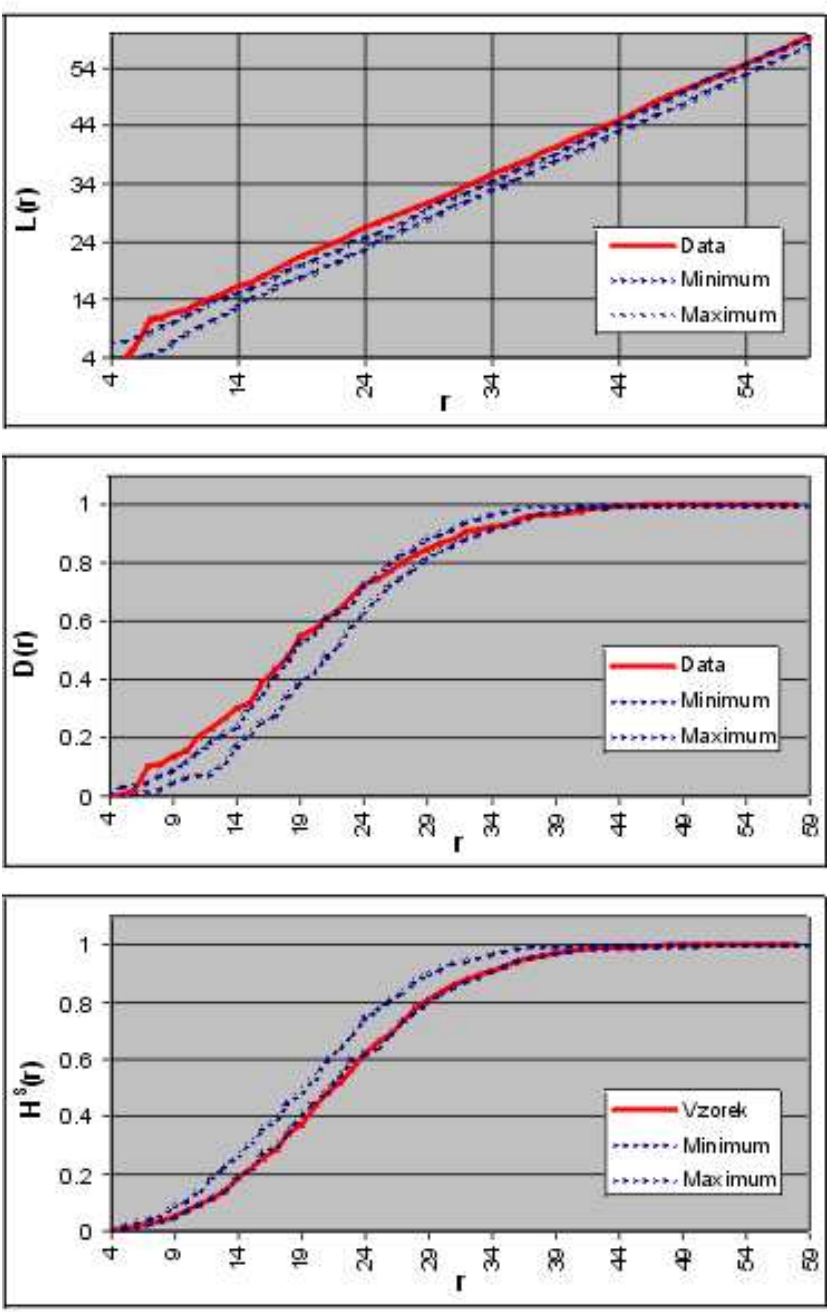

Fig. 7. The diagrams indicate values of $L-$ function $L(\cdot)$, the nearest neighbour distribution function $D(\cdot)$ and the spherical contact distribution function $H^{S}(\cdot)$ for pores of diameter equal to 4-6 $\mu \mathrm{m}$. The starting value of $r$ corresponds to minimum of the theoretical distance between the centroids of the smallest pores (in this case, 4).

It must be noted that the relative velocity of particles versus the surrounding environment (plasma + air) is not constant. The whole process is very dynamic and fluctuations of plasma parameters are at a time scale high enough to have an influence on plasma volume occupied in the same moment by tens of particles or more. The microstructure of the final coating contains structural features inherited from the "history" of individual particles in the plasma jet.
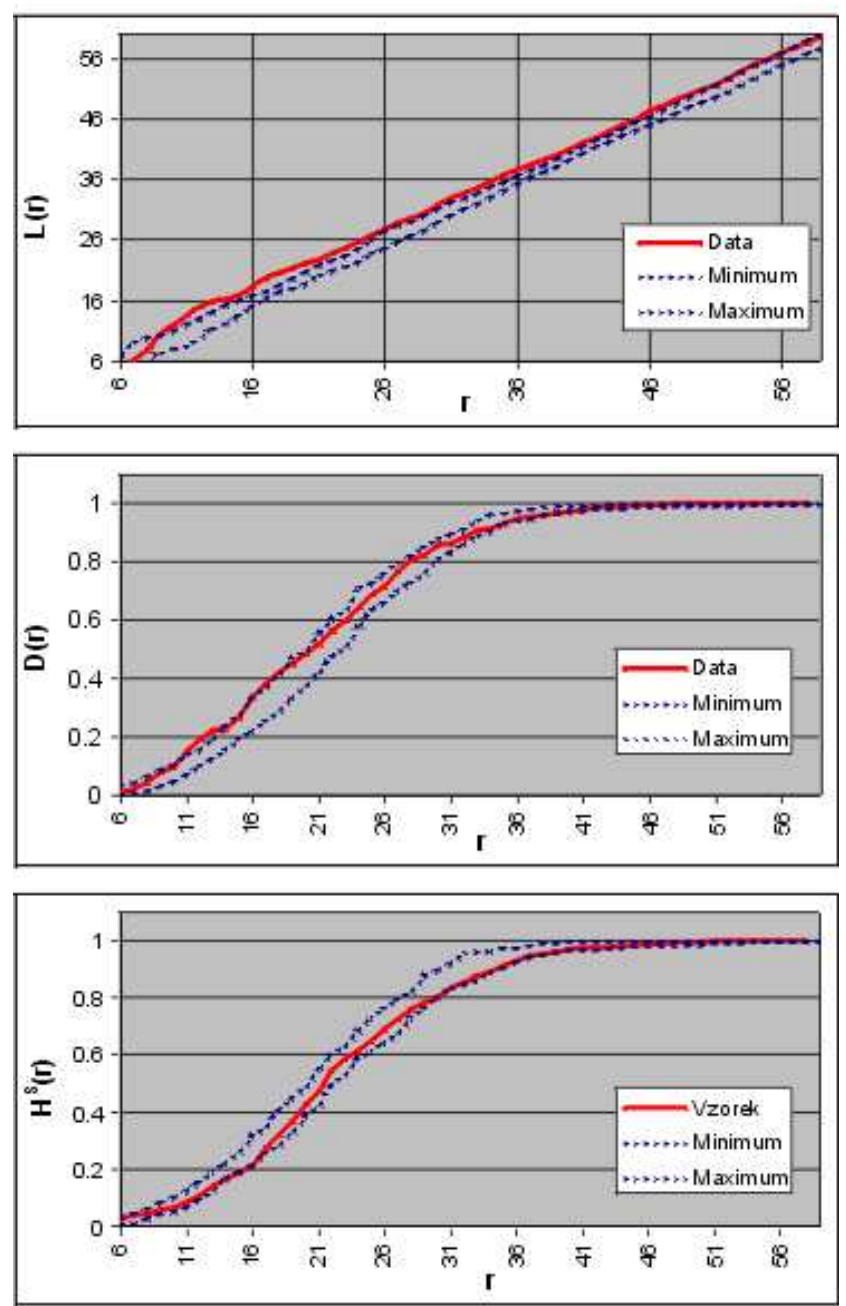

Fig. 8. The diagrams indicate values of $L$-function $L(\cdot)$, the nearest neighbour distribution function $D(\cdot)$ and the spherical contact distribution function $H^{S}(\cdot)$ for pores of diameter equal to 6-10 $\mu \mathrm{m}$. The starting value of $r$ corresponds to minimum of the theoretical distance between the centroids of the smallest pores (in this case, 6).

The present paper is based on data gained from microstructure on a high level of characterisation ability. Precise alignment of the in-flight behaviour to our microstructural data is behind the possibility of the present state of the art. But, as was documented earlier (Ctibor et al., 2003), there exists clustering of special structural features in the coating. Mild clustering of globular pores is supported by the results of the present paper. 

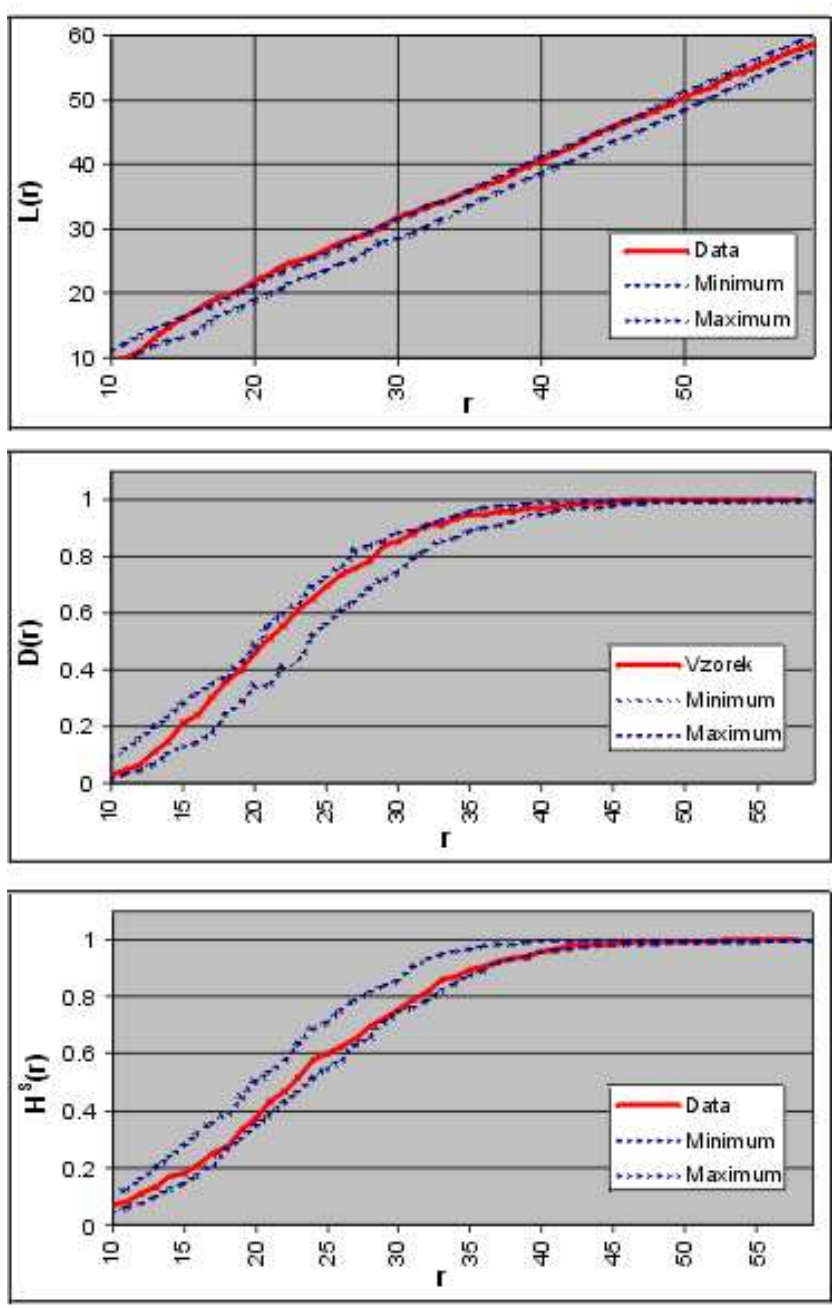

Fig. 9. The diagrams indicate values of $L$-function $L(\cdot)$, the nearest neighbour distribution function $D(\cdot)$ and the spherical contact distribution function $H^{S}(\cdot)$ for pores of diameter equal to 10-20 $\mu \mathrm{m}$. The starting value of $r$ corresponds to minimum of the theoretical distance between the centroids of the smallest pores (in this case, 10).

\section{ACKNOWLEDGEMENTS}

The research was supported by grants GAČR 201/03/0946 and MSM113200008. The autors wish to thank to their referees for helpful suggestions and to $O$. Roussel, A. Tricoire (E.N.S.I.L. Limoges, France) for their help with metallography and microscopy.

\section{REFERENCES}

Baddeley AJ, Gill RD (1997). Kaplan-Meier estimators of distance distributions for spatial point processes. Ann Stat 25:263-92.

Ctibor P, Chráska P, Roussel O, Tricoire A (2002). $3 \mathrm{D}$ reconstruction of structure of plasma spray deposits. Proceedings from the $1^{\text {st }}$ International Surface Engineering Congress and the $13^{\text {th }}$ IFHTSE Congress, Columbus, Ohio, 357-60.

Ctibor P, Roussel O, Tricoire A (2003). Unmelted particles in plasma sprayed coatings. J Eur Ceram Soc 23:29939.

Moller J, Waagepetersen RP (2003). Statistical Inference and Simulation for Spatial Point Processes. Boca Raton: Chapman \& Hall/CRC.

Ohser J, Mücklich F (2000). Statistical Analysis of Microstructures in Materials Science. New York: Wiley.

Olympus MicroImage 2, Reference Guide (1999).

Serra J (1982). Image analysis and mathematical morphology. London: Academic Press.

Soille P (1999). Morphological Image Analysis. Berlin: Springer.

Stoyan D, Kendall WS, Mecke J (1995). Stochastic geometry and its applications (second edition). New York: Wiley.

Tewari A, Gokhale AM (2000). Efficient estimation of number density in opaque material microstructures: the large area disector. J Microsc 200:277-83. 